\title{
Taylor dispersion of nanoparticles
}

\author{
Sandor Balog (D) Dominic A. Urban - Ana M. Milosevic • \\ Federica Crippa • Barbara Rothen-Rutishauser • \\ Alke Petri-Fink
}

\begin{abstract}
The ability to detect and accurately characterize particles is required by many fields of nanotechnology, including materials science, nanotoxicology, and nanomedicine. Among the most relevant physicochemical properties of nanoparticles, size and the related surface-to-volume ratio are fundamental ones. Taylor dispersion combines three independent phenomena to determine particle size: optical extinction, translational diffusion, and sheer-enhanced dispersion of nanoparticles subjected to a steady laminar flow. The interplay of these defines the apparent size. Considering that particles in fact are never truly uniform nor monodisperse, we rigorously address particle polydispersity and calculate the apparent particle size measured by Taylor dispersion analysis. We conducted case studies addressing aqueous suspensions of model particles and large-scaleproduced "industrial" particles of both academic and commercial interest of various core materials and sizes, ranging from 15 to $100 \mathrm{~nm}$. A comparison with particle
\end{abstract}

Sandor Balog and Dominic A. Urban contributed equally.

S. Balog $(\bowtie) \cdot$ D. A. Urban · A. M. Milosevic · F. Crippa B. Rothen-Rutishauser · A. Petri-Fink

Adolphe Merkle Institute, University of Fribourg, Chemin des Verdiers 4, 1700 Fribourg, Switzerland

e-mail: sandor.balog@unifr.ch

A. Petri-Fink

Chemistry Department, University of Fribourg, Chemin du Musée 9, 1700 Fribourg, Switzerland sizes determined by transmission electron microscopy confirms that our approach is model-independent, nonparametric, and of general validity that provides an accurate account of size polydispersity - independently on the shape of the size distribution and without any assumption required a priori.

Keywords Nanoparticle · Nanoparticle size characterization $\cdot$ Nanopowder $\cdot$ Polydispersity $\cdot$ Taylor dispersion

\section{Introduction}

Let it be beneficial or adverse, most often it is the size that grants unique physicochemical properties to nanoparticles (NPs). Accurate characterization is necessary, and over the last decade many methods have been applied for characterizing the size of NPs (Allen 1990; Borsali and Pecora 2008), including dynamic light scattering (DLS), fluorescence correlation spectroscopy, UV-vis spectroscopy, electron microscopy, particle tracking, atomic force microscopy, field-flow-fractionation, and analytical ultracentrifugation. Most laboratories are familiar with the majority of these techniques and their merit is indisputable. However, depending on the material, each method can reach its limits in a number of situations, such as the case of a highly heterogeneous sample or the presence of a complex multicomponent matrix (Laborda et al. 2016; Bolea et al. 2016; Moore et al. 2015) For example, fluorescence correlation spectroscopy requires dye labeling 
and UV-vis spectroscopy is essentially limited to plasmonic NPs. Standard electron microscopy is inherently an ex situ approach and generally cannot describe "soft" features - such as a polymer shell—due to either poor contrast or simply the lack of a liquid environment. Particle tracking analysis, similarly to electron microscopy, is an alternative that is able to provide numberaveraged particle size distribution, but unfortunately, it is unable to reliably detect and quantify particles smaller than $10-15 \mathrm{~nm}$ - even with strong scattering contrast, such as the one offered by gold (Filipe et al. 2010; Hawe et al. 2010). While DLS does have the sensitivity that particle tracking and electron microscopy cannot offer, it is well known that this very sensitivity becomes a serious shortcoming when samples are heterogeneous, for the capacity of measuring the true value, and the reproducibility are both prone to severely suffer from nonpurified samples. However, nowadays, to reach an unbiased verdict about the definition of poorly purified is far from trivial. Given the ever-growing complexity of suspensions of NPs - which by now come in nearly any sizes and shapes, with nontrivial composition and multifunctionalities - there are countless situations where samples cannot be fractionated or purified furthereven if it is desired for the subsequent characterization. In fact, many materials from academic and industrial labs are characterized in the very state received, preferably by using a non-destructive method.

Taylor dispersion analysis (TDA) is an in situ and non-destructive experimental technique determining the translational diffusion coefficient from a very small sample volume (from nanoliters to microliters), and the equivalent hydrodynamic radius is calculated via the Stokes-Einstein equation (Einstein 1905, 1906, 1911). Although TDA is not new, the phenomenon and the related method were described already in the 1950s (Aris 1956; Taylor 1953, 1954), it has been recently reintroduced to measuring the size of NPs (Cipelletti et al. 2014; Biron et al. 2014; Cipelletti et al. 2015; Biron et al. 2015; d'Orlye et al. 2008; Varenne et al. 2008; Hawe et al. 2011; Hulse et al. 2011; Oukacine et al. 2015; Morel et al. 2015; Pyell et al. 2015; Jalil et al. 2015; Wuelfing et al. 1999; Templeton et al. 1999). Traditionally, TDA was applied to observe chemical reactions (Chamieh et al. 2015; Biron et al. 2015; Cotett 2010) and characterize small molecules, proteins, and macro molecules (Bello et al. 1994; Rezzonico et al. 1994; Belongia and Baygents 1997; Chamieh et al. 2012; Oukacine et al. 2012;
Cipelletti et al. 2014, Biron et al. 2014; Cipelletti et al. 2015; Biron et al. 2015; Cotett 2010; d'Orlye et al. 2008; Varenne et al. 2008, Goodall 2010; Hawe et al. 2011; Hulse et al. 2011; Hulse and Forbes 2011; Oukacine et al. 2015; Morel et al. 2015; Pyell et al. 2015; Jalil et al. 2015; Saux and Cottet 2008; Wuelfing et al. 1999; Templeton et al. 1999). Lately TDA analysis has been used to characterize moderately polydisperse polymers and their mixtures (Cipelletti et al. 2014; Biron et al. 2014; Cipelletti et al. 2015; Biron et al. 2015). Particles - and especially inorganic NPs - are nonetheless quite different from small molecules, proteins, and polymers in several aspects, which actually renders them to be a quite remarkable class of materials (Giner-Casares et al. 2016; Henriksen-Lacey et al. 2016). First, unlike molecules and proteins, NPs are never truly uniform in shape or monodisperse in size. Second, the optical extinction of a given NP is a function of its size, and depending on material and the wavelength of observation, optical extinction may be dominated by either absorption or scattering.

To the best of our knowledge, the literature neither provides theoretical basis nor describes any TDA experiment taking into account the polydispersity of inorganic NPs. Therefore, here we focus on deriving an analytical approach that interprets and analyses TDA spectra from polydisperse NPs. Our primary interest is a model-independent and nonparametric description of general validity. Independently of the true shape of the size distribution - and without any assumption required a priori-our approach provides an accurate account of size polydispersity and quantifies the apparent average size determined by TDA.

After validating the approach by determining the size of a well-known protein, we investigated aqueous suspensions of dielectric and metallic NPs of various sizes. The selected NPs are model nanoparticles of academic interest (superparamagnetic iron oxide NPs (SPIONs), gold ( $\mathrm{Au}) \mathrm{NPs}$, and silica $\left(\mathrm{SiO}_{2}\right)$ NPs synthesized by us) and particles with considerable commercial interest and produced on industrial scale (zinc oxide $(\mathrm{ZnO})$, and titania $\left(\mathrm{TiO}_{2}\right)$ nanopowders, and copper $(\mathrm{Cu})$ suspensions). While $\mathrm{Cu}$ suspensions are used, e.g., for wood impregnation, SPIONs and Au NPs are model particles studied in relation to biomedical applications, and $\mathrm{SiO}_{2}$ NPs are widely found in food and agricultural technologies. $\mathrm{TiO}_{2}$ is the most widely used white pigment, for example in toothpastes, and it sees a growing demand as 
a photocatalyst. The most known application of $\mathrm{ZnO}$ produced also in multi-ton volumes - is perhaps sun protection in cosmetic products.

\section{Materials and methods}

SPIONs synthesis The iron oxide nanoparticles were synthetized by thermal decomposition, based on the method described by Park et al. (2004) (An et al. 2004). Iron oleate complex - obtained by reacting iron chloride $\left(\mathrm{FeCl}_{3} \cdot 6 \mathrm{H}_{2} \mathrm{O}\right)$ (Sigma-Aldrich, St. Louis, USA) and sodium oleate (Sigma-Aldrich, Sl. Louis, USA)was heated to and kept at $320{ }^{\circ} \mathrm{C}$ for $30 \mathrm{~min}$ in the presence of oleic acid (Sigma-Aldrich, St. Louis, USA). After the formation of particles, the solution was cooled down rapidly to room temperature and was purified via centrifugation. The oleic acid stabilized NPs were transferred to hexane and were stored at $4{ }^{\circ} \mathrm{C}$. To transfer the SPIONs to water, the oleic acid was replaced by citric acid (Sigma-Aldrich, St. Louis, USA) following the procedure described elsewhere (Lattuada and Hatton 2007).

Citrate-stabilized Au NPs First small seed Au NPs (approximately $7 \mathrm{~nm}$ in radius) were synthesized based on the method described by Turkevich et al. (1951) (Stevenson et al. 1951). A $500 \mathrm{~mL}(0.5 \mathrm{mmol} / \mathrm{L})$ chloroauric acid (HAuCl4) (Sigma-Aldrich, St. Louis, USA) solution was brought to boil for approximately 5-10 $\mathrm{min}$, prior to the addition of a $25 \mathrm{~mL}(34 \mathrm{mmol} / \mathrm{L})$ sodium citrate (Sigma-Aldrich, St. Louis, USA) solution. The mixture was kept at boiling temperature for additional 15-20 min, during which time the color shift from yellow to black to wine red can be observed, before the mixture was removed from heat and left to cool to room temperature for 2-3 h. Next, following the work of Brown et al. (2000) (Walter et al. 2000), a $600 \mathrm{~mL}$ aqueous solution of HAuCL4 $(0.25 \mathrm{mM})$ and hydroxylamine hydrochloride (Sigma-Aldrich, St. Louis, USA) $(1.96 \mathrm{mM})$ was prepared in a glass bottle and homogenized with a magnetic stirrer. The previously described seed particles were used as gold seed suspension $(1.2 \mathrm{~mL})$ and added to the mixture. The mixture was stirred constantly for approximately $20 \mathrm{~min}$, during which time a color shift could be observed. The resulting NPs were stabilized by the addition of $7.14 \mathrm{mg}$ of sodium citrate. Purification of the NPs was done by centrifugation (4000 rpm for $15 \mathrm{~min}$ ) and re- dispersion in MilliQ water (Millipore purification system, Bedford, MA, USA).

$\mathrm{SiO}_{2} \mathrm{NPS}$ The $\mathrm{SiO}_{2} \mathrm{NPs}$ were synthesized by following a modified procedure of the Stöber synthesis, described by Giesche et al. (1994). A mixture of ethanol, MilliQ water, and ammonia (VWR, Dietikon, Switzerland) were transferred to a round bottom flask, heated with the help of an oil bath, and stirred with a magnetic stirrer. TEOS was transferred rapidly to the round bottom flask and continuously stirred for several hours. Next, the ethanol was removed from the suspension by evaporation under reduced atmosphere. The particles were dialyzed in water for 5 days.

$\mathrm{ZnO}$ NPs Zinc oxide NPs were obtained in powder form (Sigma-Aldrich 721077). The sample was prepared by dispersing the powder in water, which was followed by $10 \mathrm{~min}$ of sonication and subsequent dilution.

$\mathrm{TiO}_{2}$ NPs Titanium dioxide NPs were obtained in powder form (Sigma-Aldrich, Degussa, P25). The sample was prepared by dispersing the powder in water, which was followed by $10 \mathrm{~min}$ of sonication and subsequent dilution.

$\mathrm{Cu} N P s$ The commercially available copper carbonate NPs were used as received without further modification. For reasons of confidentiality and trade secrecy, the content and synthetic route was not disclosed.

Taylor dispersion Taylograms were collected at constant temperature $\left(25^{\circ} \mathrm{C}\right)$. The initial concentrations of the samples - quantified as the number of particles per volume-were estimated by using a numberaveraged mean radius, assuming spherical particles (Supporting Information, The initial particle concentrations of the samples). Time-resolved extinction was followed by an ActiPix D100 UV-Vis area imaging detector (Paraytec, York, UK, $20 \mathrm{~Hz}$ sample rate). Samples were injected into a fused silica capillary $(74.5 \mu \mathrm{m}$ inner diameter, Polymicro Technologies, Phoenix, USA) under continuous flow conditions, using a capillary electrophoresis injection system (Prince $560 \mathrm{CE}$ Autosampler, Prince Technologies B.V., Netherlands). For the $\mathrm{SiO}_{2}$ NPs, BSA, and $\mathrm{Cu}$ NPs, the running buffer was MilliQ water. To 
prevent NP-capillary wall interactions, a $0.001 \%(w /$ v) TWEEN®20/water (Sigma-Aldrich, St. Louis, USA) solution was used as running buffer for the $\mathrm{Au}$ NPs and SPIONs. Depending on the optical extinction of a sample, varying injection volumes were used. After sample injection, a pressure of $70 \mathrm{mbar}$ was applied to drive the samples through the capillary. The total capillary length was $130 \mathrm{~cm}$, with the length to the end of the first window being $45 \mathrm{~cm}$ and the length to the end of the second window being $85 \mathrm{~cm}$. Both windows had a width of approximately $1 \mathrm{~cm}$, and a band-pass filter (10 nm FWHM) coupled with a neutral density filter (10\% transmission) were used (Edmund Optics, York, UK). The center wavelength of the band-pass filters were 520, 520, 214, 280, 280, and $400 \mathrm{~nm}$ for the SPIONs, Au NPs, SiO2 NPs, $\mathrm{ZnO}$ powder, $\mathrm{TiO} 2$ powder, and $\mathrm{Cu}$ suspension, respectively. Regarding calibration and data collection, the instrument optimizes data acquisition. To ensure that the detector response is linear, the instrument controls the intensity of illumination. The intensity values measured are corrected for dark current, and the background corresponding to the solvent is measured before each run. In order to eliminate the impact of possible fluctuations and slow variations in the intensity of illumination, reference pixels continuously monitor the intensity of illumination reaching the sample. The extinction of the particles is therefore always expected to be measured in the linear range of the response of the detector, and the taylogram is calculated via taking into account dark current, solvent background, and variations in the illumination. To improve the final signal-to-noise ratio, the taylograms were smoothed by a centered moving average algorithm before analysis (Supporting Information, The algorithm of smoothing the raw spectra, The effect of smoothing on the taylograms of NPs).

Transmission electron microscope The sample preparation for TEM analysis followed two methodologies. The $\mathrm{SiO}_{2}$ NPs and SPIONs samples were prepared by drop casting of the diluted samples onto the TEM grids. The remaining NP samples were prepared by following a method described in detail elsewhere (Michen et al. 2015; Geers et al. 2015). Briefly, the sample preparation requires drying, which often introduces artifacts, e.g., aggregation and the so-called coffee-ring effect (Michen et al. 2015; Geers et al. 2015). We prevented the onset of these artifacts by adding a water-soluble globular protein to the suspension. The protein assists in stabilizing individual particles against aggregation, mitigates substrate dewetting, fortifies Marangoni flow, and results in a less heterogeneous deposition of single particles onto the TEM grid. After sample preparation, $5 \mu \mathrm{L}$ of the solution were drop-cast on a TEM grid and allowed to dry prior to TEM analysis. All images were acquired using an FEI Tecnai Spirit TEM with a Veleta $2048 \times 2048$ CCD camera at $80 \mathrm{kV}$. The image analysis was performed using ImageJ.

\section{Theory}

Uniform particles The left side of Fig. 1 illustrates the fundamental mechanisms behind dispersing NPs in a laminar flow. Briefly, one injects the sample into a thin capillary, and the combination of pressuredriven advection and translational diffusion disperses the particles. The sample volume injected ranges from a few nanoliters to microliters, depending on the experimental setup. The dispersion of the injected particles is observed through their optical extinction, and its temporal evolution carries information about translational diffusion, and thus, about particle size. Under the most frequently used conditions, the normalized optical extinction recorded as a function of time - usually referred to as taylogramcorresponding to uniform molecules, proteins, or particles of hydrodynamic radius $r$ can be written as follows (Aris 1956; Taylor 1953, 1954):

$c(t, r)=\frac{1}{\sqrt{\pi}} \frac{1}{\sqrt{\delta \cdot r \cdot t}} e^{-\frac{\left(t-t_{0}\right)^{2}}{\delta \cdot r \cdot t}}$

$\delta=\frac{\pi \eta \mathrm{Y}^{2}}{2 k_{\mathrm{B}} T}$, and $Y$ is the capillary radius, $\eta$ the viscosity of the fluid, $T$ the temperature, and $k_{\mathrm{B}}$ the Boltzmann constant. $t_{0} \equiv x / v$ is the so-called residence time, which is defined by the distance between detection and injection points $\left(x \equiv x_{\mathrm{det}}-x_{\mathrm{inj}}\right)$ and the velocity of the flow (v) averaged over the cross section of the capillary. The analysis begins with determining the first three central moments of a taylogram. It is easy to calculate that for uniform NPs the mean value is the following: 


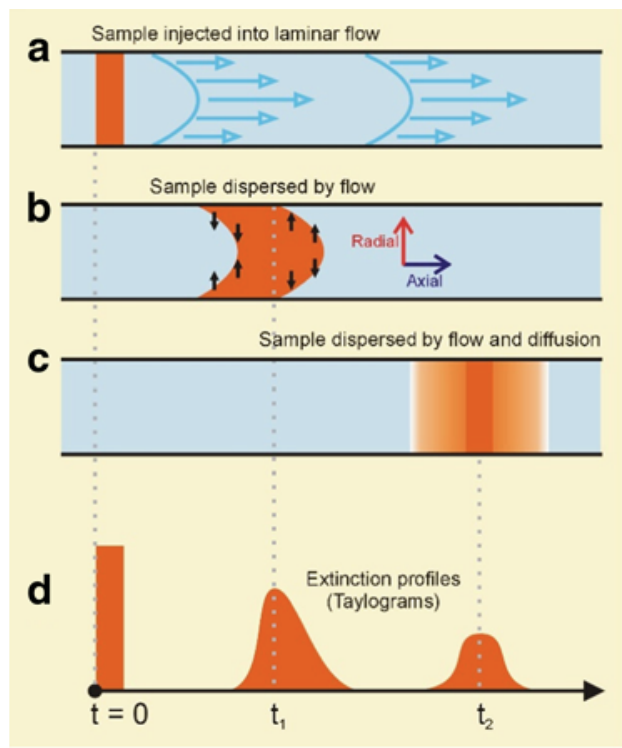

Fig. 1 Left: (a) The sample is injected into a cylindrical capillary supporting a pressure-driven stationary laminar flow. The fluid moves in concentric layers with a parabolic velocity profile (Poiseuille flow). (b) At short residence times advection dominates, and the concentration of the sample mostly follows the flow profile. However, owing to concentration gradients caused by advection, the particles at the front of the dispersion band migrate towards the walls of the capillary, while particles at the back migrate towards the center. This is referred to as radial mixing, and the rate of migration is defined by the translational diffusion of the NPs. Compared to advection, the contribution of axial diffusion to the dispersion parallel to the flow is negligible. (c) Eventually, radial mixing homogenizes the concentration of the sample

$M_{\mathrm{t}} \equiv \int_{0}^{\infty} t \cdot c(t, r) \mathrm{dt}=t_{0}+\frac{\delta \cdot r}{2}$

the variance

$V_{\mathrm{t}} \equiv \int_{0}^{\infty}\left(t-M_{\mathrm{t}}\right)^{2} \cdot c(t, r) \mathrm{dt}=\frac{\delta \cdot r}{2}\left(t_{0}+\delta \cdot r\right)$

and the skewness

$S_{\mathrm{t}} \equiv \frac{1}{V_{\mathrm{t}^{\frac{3}{2}}}} \int_{0}^{\infty}\left(t-M_{\mathrm{t}}\right)^{3} \cdot c(t, r)$

$\mathrm{dt}=\frac{\delta^{2} \cdot r^{2}\left(3 t_{0}+4 \cdot \delta \cdot r\right)}{\sqrt{2}\left(\delta \cdot r \cdot\left(t_{0}+\delta \cdot r\right)\right)^{3 / 2}}$

The right side of Fig. 1 illustrates how these moments describe the shape of the taylogram.

Polydisperse particles We extend Eq. (1) to polydisperse NPs by considering the size-dependent optical

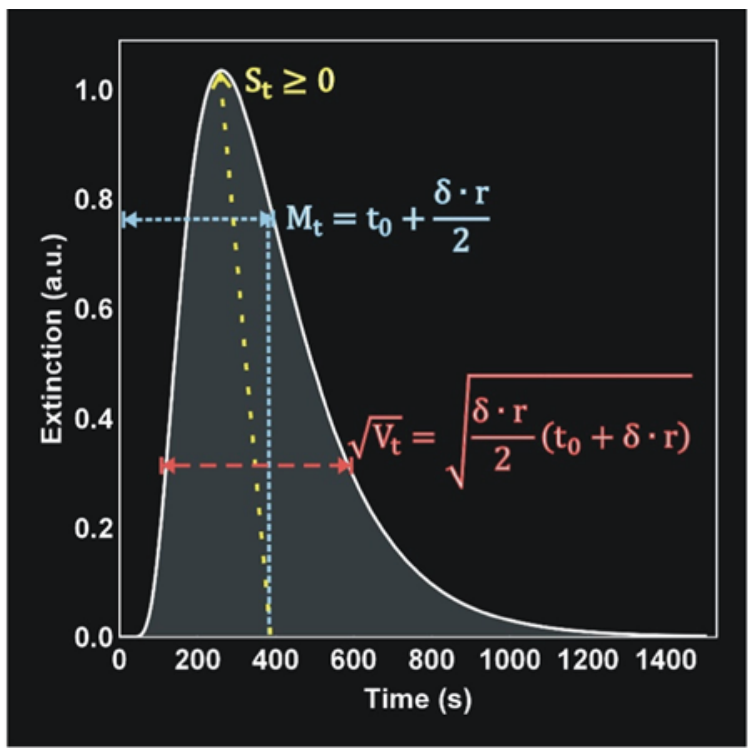

across the cross section of the capillary, while the axial profile remains non-homogenous and its shape carries information about the translational diffusion coefficient and particle size (information is given on the right side figure). (d) The corresponding extinction profiles are illustrated: Injection leaves a sample profile with a finite width, which extends further due to advection and diffusion. Right: Depicting the first three central moments of a taylogram of uniform particles: The mean $\left(M_{\mathrm{t}}\right)$ and the variance $\left(V_{\mathrm{t}}\right)$ describes the location and width, while the skewness $\left(S_{\mathrm{t}}\right)$ describes the symmetry of the shape. A taylogram cannot have a negative $S_{\mathrm{t}}$ value. After sufficiently long time, the shape is close to a Gaussian, that is, $M_{\mathrm{t}} \cong t_{0}, V_{\mathrm{t}} \cong 1 / 2 \cdot \delta \cdot r \cdot t_{0}$, and $S_{\mathrm{t}} \cong 0$

extinction $\mu(r, \lambda)$ of a particle at the detection wavelength $\lambda$. Given that the appearance of each particle is weighted by its optical extinction, the taylogram of polydisperse NPs is expressed as:

$C(t)=\frac{\int_{0}^{\infty} P(r) \mu(r, \lambda) c(t, r) \mathrm{dr}}{\int_{0}^{\infty} P(r) \mu(r, \lambda) \mathrm{dr}}$

where $P(r)$ is the probability density distribution of the particle radius, and thus $P(\mathrm{r}) \times \mathrm{dr}$ quantifies the probability that the radius is found in dr interval about $r$. Equation (5) is of general validity, where $C$ represents the optical extinction as function of time, and defined as the taylogram from polydisperse particles. A consequence of Eq. (5) is that in case of polydispersity, the taylogram is in fact never a single Gaussian (Chamieh et al. 2012; Oukacine et al. 2012). Therefore, interpreting TDA spectra with one Gaussian is therefore 
an approximation that is only suitable at sufficiently low polydispersity. Furthermore, the exact analytical form of Eq. (5) is dependent on $P(r)$; hence, fitting an analytic function requires a parametric model distribution that adequately describes the true particle size distribution. While models are practical in certain situations (Geers et al. 2016; Rodriguez-Lorenzo et al. 2016), they are not always available or represent real scenarios, and thus the true shape of $P(r)$ frequently remains unknown. To circumvent this limitation, we apply here a more general, model-independent, and accurate non-parametric description. The rationale behind this approach is straightforward: due to the role of diffusion in the dispersion process, a taylogram may be regarded as a probability distribution, and thus, we use the concept of statistical moments to describe the spectra (Chamieh et al. 2012; Oukacine et al. 2012; Cottet et al. 2007; Martin et al. 2007). Given that the optical extinction of NPs is a power function of the radius, $\mu(r, \lambda) \propto r^{\mathrm{n}}$ (Quinten $2011)$, it can be shown that the apparent radius $(R)$ of polydisperse NPs measured by TDA is as follows:

$R \equiv \frac{\left\langle r^{n+1}\right\rangle}{\left\langle r^{n}\right\rangle}$

where $r^{n}$ is the $n^{\text {th }}$ raw moment of the particle size distribution

$$
\left\langle r^{n}\right\rangle \equiv \int_{0}^{\infty} P(r) \cdot r^{n} \mathrm{dr}
$$

Regarding Eq. (6), the necessary calculations, are given in the Supporting Information (Apparent radius of polydisperse NPs determined by the statistical moments). The value of the apparent radius $(R)$ also depends on whether absorption or scattering dominates the optical extinction. Numerical computations (Supporting Information, Optical extinction of spherical nanoparticles via Mie calculations) show that absorption and scattering of NPs scales approximately as $r^{3}$ and $r^{6}$ on a wide but finite range. The upper limit of this range is a function of the wavelength and the refractive indices of the particle and suspending liquid. Considering that, the size distribution of NPs is always limited to a finite range, this scaling between size an optical extinction can generally be reached by choosing appropriately the wavelength of illumination and detection. For dielectric NPs, absorption in the visible range is negligible, and thus, $n=6$. For most metallic NPs, absorption dominates, and $n=3$. The meaning of Eq. (6) becomes clearer when polydispersity is moderate. Then, the apparent radius determined by TDA is the following:

$R \cong(r)+n \cdot \frac{\sigma^{2}}{\langle r\rangle}$

where $\langle r\rangle$ is the mean radius and $\sigma \equiv \sqrt{\left\langle r^{2}\right\rangle-\langle r\rangle^{2}}$ is defined as the standard deviation of the particle size distribution. Thus, the apparent radius characterized by TDA is increasing with the variance of the particle size distribution, and the proportionality constant is defined by the dominant "mode" of optical extinction. Figure 2 shows that $R$ is always bigger than $r$ when $\sigma>0$, and increases with polydispersity. $R$ is considerably larger than $r$ when the polydispersity is higher than $25 \%$.

\section{Results and discussion}

$B S A$ protein Using the approach of statistical moments, we first characterize the hydrodynamic radius of bovine serum albumin (BSA). BSA is a well-defined standard macromolecule and found in numerous applications regarding biochemical assays. BSA is a globular protein with a molecular weight of $66.8 \mathrm{kDa}$, and its hydrodynamic radius is reported to be between 3.3 and $4.3 \mathrm{~nm}$ (Hawe et al. 2011; Hulse et al. 2011). Figure 3 shows two taylograms recorded at two residence times from an aqueous solution of BSA. As explained later, two residence times are used to minimize the impact of a noninstantons injection.

The statistical moments are calculated as defined in Eqs. (2-4). The radius $r$ and the exact residence time $t_{0}$ - set by the velocity of the laminar flow and the distance between injection and detection points-are

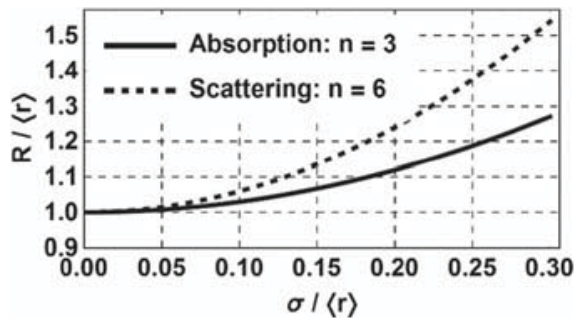

Fig. 2 The theoretical ratio of the apparent radius and the mean radius as a (quadratic) function of polydispersity. The curves are calculated via Eq. (7). 


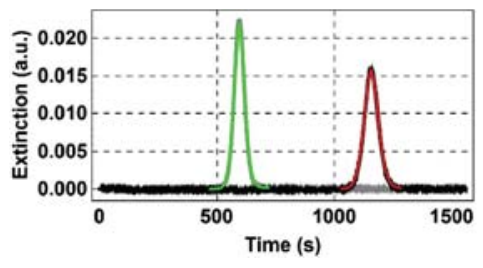

Fig. 3 Left: Taylograms recorded from an aqueous solution of BSA (detection wavelength is $214 \mathrm{~nm}$ ). The intervals carrying the information about particle size are highlighted in color. Right: The variance of the taylograms determined at two residence times

determined by solving Eqs. 2 and 3 simultaneously. The skewness is small, indicating that the taylograms are highly symmetric, and thus, both corresponds well to a Gaussian profile. Regarding the variance, it is important to mention that the experimentally determined width of the taylogram is generally the sum of two terms, $V(t)=$ $V_{\mathrm{t}}(t)+\Delta \mathrm{V}$, where the second term represents the initial width of the sample and accounts for a systematic deviation due to the non-instantaneous injection. To minimize this systematic error, one frequently uses more than one detection points (Chamieh et al. 2012; Oukacine et al. 2012). Given that $V\left(t_{2}\right)-V\left(t_{1}\right)=V_{\mathrm{t}}\left(t_{2}\right)$ $-V_{\mathbf{t}}\left(t_{1}\right), \Delta V$ can be eliminated, and one obtains that the radius is given by the following:

$r=\frac{2}{\delta} \frac{V\left(t_{2}\right)-V\left(t_{1}\right)}{t_{2}-t_{1}}$

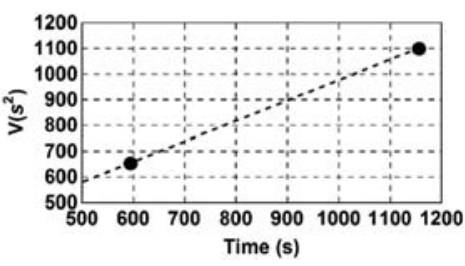

(symbols) and the best linear fit (dashed line). The radius is determined from the slope of this line (Eq. (12)). The non-zero intercept of this line results from the initial width of the taylogram due to the non-instantaneous sample injection (see text below)

The analysis of the statistical moments combined with Eq. (9) determines a hydrodynamic radius of $3.4 \mathrm{~nm}$, which is in excellent agreement with the values reported in the literature (Hawe et al. 2011; Hulse et al. 2011).

Model NPS Next, we use this approach to characterize three different model NPs. The taylograms of SPIONs, $\mathrm{Au}$ NPs, and $\mathrm{SiO}_{2}$ NPs are symmetric, which indicates that their shape is near to a Gaussian (Fig. 4). The analysis of the moments is straightforward and Table 1 lists the apparent radii. Given that TEM characterization can be used to estimate the size distribution of these particles, the NPs were also imaged by electron microscopy and the micrographs were analyzed in terms of mean $(r)$ and standard deviation $(\sigma)$ (Table 1). The standard deviation was estimated by counting particles, where the exact number of particle counts varied from
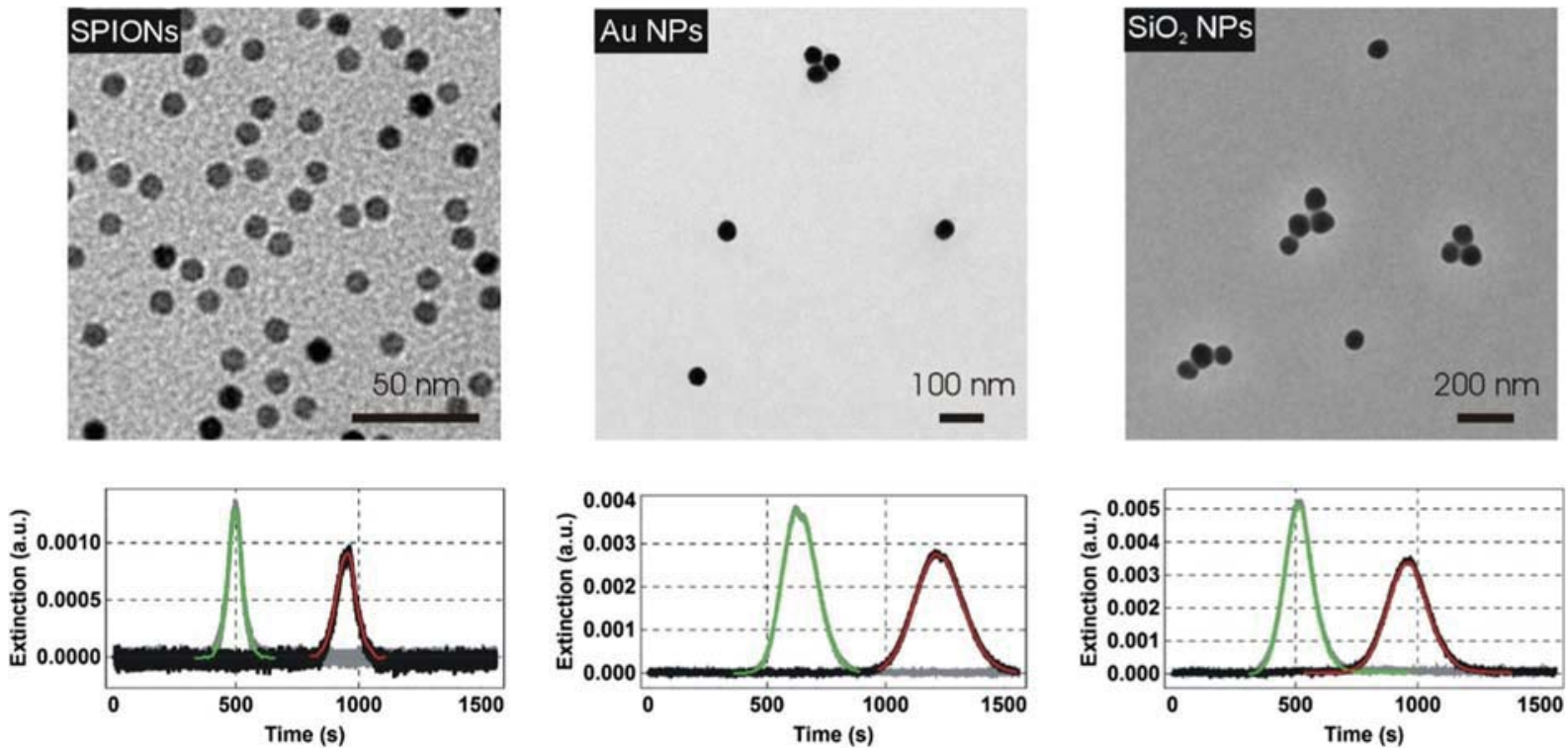

Fig. 4 TEM micrographs and taylograms of the model NPs. The intervals carrying the information about particle size are highlighted in color 
Table 1 Radii of the model NPs determined by TDA in one single measurement (apparent radius $R$ ) and TEM (orientation-averaged Feret radius (Merkus 2009))

\begin{tabular}{lrl}
\hline & $\begin{array}{l}\text { TDA } \\
(\mathrm{nm})\end{array}$ & $\begin{array}{l}\text { TEM } \\
(\text { mean } \pm \text { STD }) \\
(\mathrm{nm})\end{array}$ \\
\hline $\mathrm{SPIONs}$ & 7.6 & $6.7 \pm 1.1$ \\
$\mathrm{Au} \mathrm{NPs}$ & 33.8 & $28.0 \pm 4.7$ \\
$\mathrm{SiO}_{2} \mathrm{NPs}$ & 44.0 & $39.3 \pm 6.2$ \\
\hline
\end{tabular}

sample to sample, and was between 300 and 2000. In contrast to TEM, Taylor dispersion is an ensemble method that probes milliards of NPs simultaneously in a single measurement. Relying on Mie theory (Quinten 2011), it can be shown that at the detection wavelength chosen, the optical extinction of SPIONs and Au NPs is dominated by absorption, while for $\mathrm{SiO}_{2} \mathrm{NPs}$ by scattering (Supporting Information, Optical extinction of spherical nanoparticles via Mie calculations). Equation 8 means that it matters whether the optical extinction of the NPs is dominated by absorption or scattering, for it defines the apparent hydrodynamic radius measured by TDA. This is what we took into account when testing the consistency between the experimentally obtained TDA results and the central-moment analysis of polydisperse NPs, summarized in Eqs. (7) and (8). Combining the results of Mie calculations $(n)$ and TEM analysis ( $r$ and $\sigma$ ), we predicted $R$ independently via Eq. (8).
The apparent radii predicted this way were 7.2, 30.4, and $45.2 \mathrm{~nm}$ for SPIONs, $\mathrm{Au}$ NPs, and $\mathrm{SiO}_{2} \mathrm{NPs}$, respectively. The agreement between theory and experiment is within $10 \%$, which supports the validity of our analysis.

Large-scale produced "industrial" NPs Finally, we use the same approach to characterize three different commercially available NPs by Taylor dispersion. The industrial particles, $\mathrm{ZnO}, \mathrm{TiO}_{2}$, and $\mathrm{Cu} \mathrm{NPs}$, are produced on a large scale. The TEM micrographs show that they are highly heterogeneous and of arbitrary shape (Figure 5). Given that their taylograms are obviously not Gaussian functions, that is, the $\mathrm{ZnO} N P s, \mathrm{TiO}_{2} \mathrm{NPs}$, and the $\mathrm{Cu}$ suspension are asymmetric and the $\mathrm{Cu}$ suspension exhibits multimodality, the advantage of using the statistical moments is evident. We must put an accent on the fact that an accurate characterization of size (distribution) of these nanomaterials is very challenging. Their characterization with perhaps the most known in situ technique, i.e., DLS, as matter of fact is meaningless without a thorough purification. Furthermore, any standard TEM analysis of such nanopowder samples requires a preparation procedure without which the TEM analysis has de facto no practical use (Michen et al. 2015; Geers et al. 2015).

Based on TEM, the retailer claims approximately $20 \mathrm{~nm}$ average primary particle size for the $\mathrm{TiO}_{2} \mathrm{NPs}$ and $\leq 40 \mathrm{~nm}$ for the $\mathrm{ZnO}$ NPs. Our TEM characterization
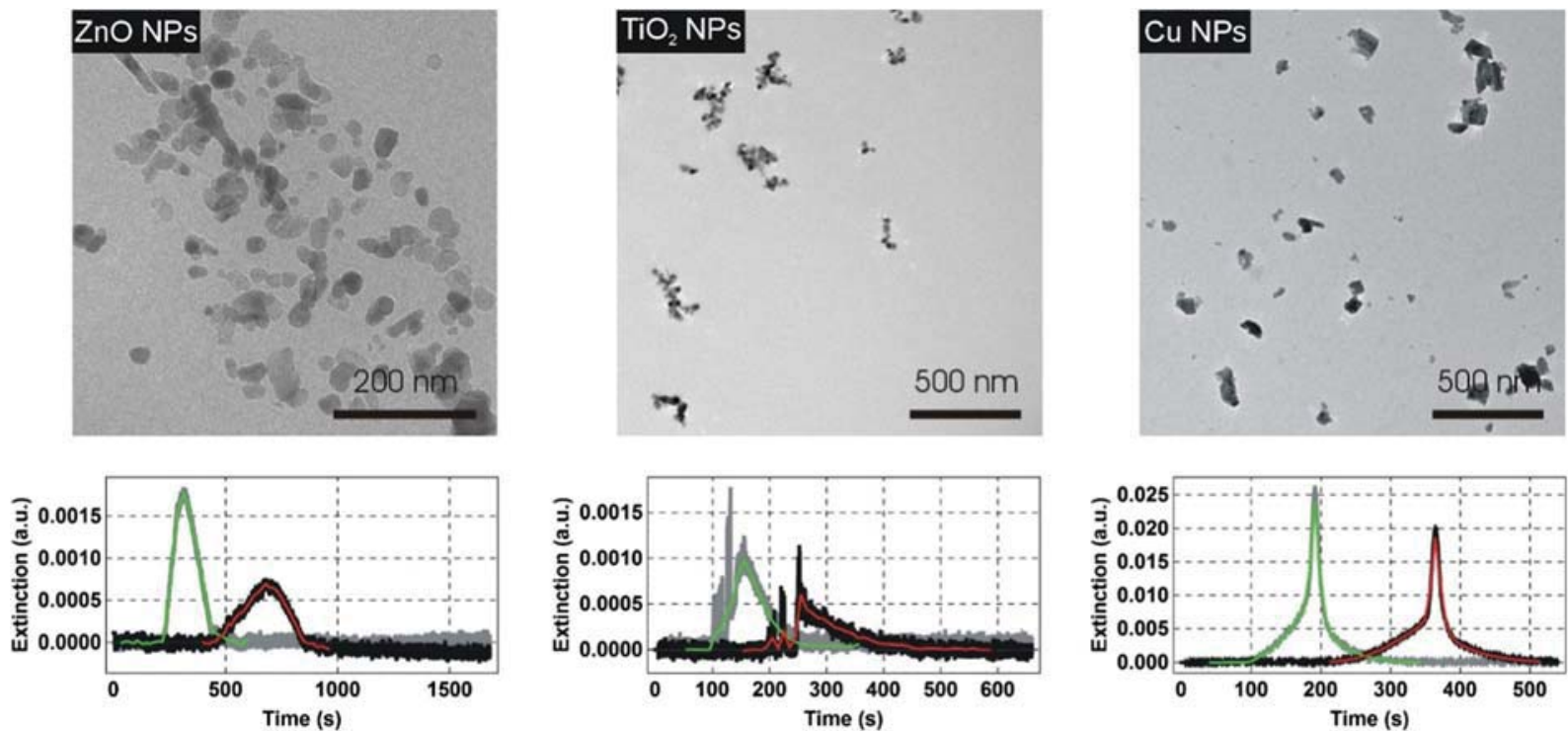

Fig. 5 TEM micrographs and taylograms of the "industrial" NPs. The intervals carrying the information about particle size are highlighted in color. Table 2 summarizes the results of the analyses 
of the industrial samples confirms high polydispersity, and the STD is comparable to the mean value. This suggests that the particle size as obtained from powder follows a long-tailed distribution or at least an exponential one. Consequently, we expected that during the TDA experiment, the influence of laminar flow and gravity may fractionate the samples to certain extent, and aggregates and agglomerates may settle in the capillary already before reaching the detection point. Regarding this issue, currently we are unable to provide detailed information but it indeed posed some difficulty: the signal-to noise ratio was low and set a limitation to extending the residence time when recording the taylograms of the $\mathrm{TiO}_{2}$ and $\mathrm{ZnO}$ NPs. Therefore, in this case, the theoretical prediction based on TEM cannot be directly compared to TDA, because TEM and TDA does not probe the same ensemble of particles of the given sample. Nonetheless, Table 2 shows that we could detect and analyze NPs of the powder samples.

The taylograms of the $\mathrm{Cu}$ NPs shows that the sample is multimodal, and a relatively narrow peak is superimposed onto a broad one. The width of this narrow peak indicates the presence of sub-nanometer particulates. Whether these are indeed particles or molecules and fragments remaining from synthesis could not be clarified by the manufacturer. Such evident multimodality points into the direction of addressing multicomponent and complex materials enhanced with NPs - such as food products - but it is well beyond the scope of the current work.

It is also worthwhile to consider what determines the concentration of nanoparticles suitable for TDA analysis. The signal is carried by the optical extinction, which is a function of the optical path length (capillary diameter), the concentration and optical extinction of single particles. Optical extinction is dependent on the particle size and its material as well as on the refractive index of the fluid. Additionally, optical extinction is also a

Table 2 Radii of the "industrial" NPs determined by TDA (apparent radius $R$ ) and TEM (orientation-averaged Feret radius (Merkus 2009))

\begin{tabular}{lll}
\hline & $\begin{array}{l}\text { TDA } \\
(\mathrm{nm})\end{array}$ & $\begin{array}{l}\text { TEM } \\
(\text { mean } \pm \mathrm{STD}) \\
(\mathrm{nm})\end{array}$ \\
\hline $\mathrm{ZnO}$ powder & 28.8 & $28.5 \pm 18.1$ \\
$\mathrm{TiO}_{2}$ powder & 50.0 & $117.5 \pm 101.4$ \\
$\mathrm{Cu}$ suspension & 33.1 & $27.9 \pm 26.8$ \\
\hline
\end{tabular}

function of the wavelength. Beside the capillary diameter, the overall volume of the observed sample is defined by the detector area, pixel size, and binning of pixels. Furthermore, the wavelength-dependent response of the detector - its noise-level and (linear) dynamic rangeand the spectral distribution of the light source may become critical factors when defining the lower limit of concentration. Accordingly, the time resolution of data acquisition must also be optimized, since a high time resolution usually results in increased noise. In addition, one must consider that with increasing residence time, the observable concentration is decreasing due to the continuous brand broadening. Therefore, given that there are many parameters that can define the range of applicable concentration for any given NP system, the limits are specific to the given sample. Nonetheless, we expect that - similarly to UV-Vis spectroscopy-these specific limits can be easily estimated and calibrated after a few runs using different concentrations.

Another aspect is the case of anisotropic particles, such as rods and spheroids. Depending on the degree of anisotropy, the transport may be considerably more complex in the presence of flow. Given that diffusion of such particles is tensorial, we anticipate that depending on the dimensions - for example, the length and aspect ratio of the particles and the diameter of the capillary - and the velocity of the fluid flow (shear stress), the rods at one point will tend to align parallel to the capillary axis. This means that - due to such orientation - the translational diffusion will become anisotropic and heterogeneous across the capillary cross section, which in fact, influences the dispersion profile. In addition, one must consider that the optical extinction of such NPs is a function of dimensions defined by at least two or three parameters, such as length and aspect ratio of a rod and the axes of a spheroid. Therefore, the optical extinction can exhibit a more complex dependence upon varying these parameters, and TDA analysis will require a more elaborate approach.

\section{Conclusion and outlook}

To summarize, we developed a new approach to analyze the Taylor dispersion spectra of nanoparticles. The use of statistical moments - which is a general, model-independent, and non-parametric description-enables the 
accurate analysis of non-Gaussian and asymmetric taylograms. Regarding NPs, this work is the first to describe rigorously the apparent size measured by TDA from polydisperse NPs. TDA analysis was complemented by TEM, and a good agreement was found between these two markedly different methods. The $\mathrm{TiO}_{2}$ powder, however, was an exception. Given that TDA analysis did not probe the large aggregates and agglomerates, TEM analysis resulted in considerably higher size. Nonetheless, unlike TEM, TDA analysis agreed better with the size given by the retailer claiming approximately $20 \mathrm{~nm}$ average primary particle size. Regarding these materials received from industry - to our knowledge - this is the first report of successful TDA characterization addressing particulate nanomaterials of such heterogeneity. Characterizing these large-scale-synthesized materials "as received" is challenging for today's analytical methods. Nonetheless, TDA was able to detect and quantify nanoscale features despite the apparent multimodality and the presence of micron-sized aggregates and agglomerates, without involving sample preparation and purification.

We believe these are the first steps for TDA analysis towards detecting and accurately quantifying particulate nanomaterials dedicated to enhance the properties of commercial products, perhaps even in multicomponent and highly heterogeneous matrices, which is relevant for the assessment of risk and safety of applications of nanomaterials.

The application of this approach for quantifying polydispersity will be addressed in another work.

Acknowledgements The authors are grateful for the financial support of the Adolphe Merkle Foundation and the University of Fribourg. SB, AMM, FC, and BRR acknowledge the financial support of the Swiss National Science Foundation through the National Centre of Competence in Research Bio-Inspired Materials.

Author contributions SB and AP-F led the study. SB wrote the manuscript through contributions of the co-authors. SB derived the theoretical approach and analyzed the taylograms. DAU and AM set up the TDA experiments and collected the taylograms. DAU and FC synthesized the Au NPs the SiO2 NPs, and the SPIONs, respectively. TEM micrographs were collected by DAU and were analyzed by DAU and SB. All authors have read and given approval to the final version of the manuscript.

\section{Compliance with ethical standards}

Conflict of interest The authors declare that they have no competing or financial interest.

\section{References}

Allen T (1990) Particle Size Measurement. Springer, Dordrecht Aris R (1956) On the dispersion of a solute in a fluid flowing through a tube. Proc R Soc Lond A Math Phys Sci 235(1200):67-77

Bello MS et al (1994) Use of taylor-aris dispersion for measurement of a solute diffusion-coefficient in thin capillaries. Science 266(5186):773-776

Belongia BM, Baygents JC (1997) Measurements on the diffusion coefficient of colloidal particles by taylor-aris dispersion. J Colloid Interface Sci 195(1):19-31

Borsali R, Pecora R Eds. (2008) Soft Matter Characterization. Springer, Dordrecht

Brown KR et al (2000) Seeding of colloidal au nanoparticle solutions. 2. Improved control of particle size and shape. Chem Mater 12(2):306-313

Chamieh J et al (2015) Monitoring biopolymer degradation by taylor dispersion analysis. Biomacromolecules 16(12):39453951

Chamieh J et al (2012) Taylor dispersion analysis with two detection points on a commercial capillary electrophoresis apparatus. J Chromatogr A 1235:174-177

Cipelletti L et al (2014) Polydispersity analysis of taylor dispersion data: the cumulant method. Anal Chem 86(13):6471-6478

Cipelletti L et al (2015) Measuring arbitrary diffusion coefficient distributions of nano-objects by taylor dispersion analysis. Anal Chem 87(16):8489-8496

Cotett H (2010) Determination of individual diffusion coefficients in evolving binary mixtures by taylor dispersion analysis: application to the monitoring of polymer reaction. Anal Chem 82:1793-1802

Cottet $\mathrm{H}$ et al (2007) Determination of dendrigraft poly-L-lysine diffusion coefficients by taylor dispersion analysis. Biomacromolecules 8(10):3235-3243

d'Orlye F et al (2008) Determination of nanoparticle diffusion coefficients by taylor dispersion analysis using a capillary electrophoresis instrument. J Chromatogr A 1204(2):226232

Einstein A (1905) The motion of elements suspended in static liquids as claimed in the molecular kinetic theory of heat. Ann Phys 17(8):549-560

Einstein A (1906) Eine neue bestimmung der moleküldimensionen. Ann Phys 324(2):289-306

Einstein A (1911) Berichtigung zu meiner arbeit: "eine neue bestimmung der moleküldimensionen". Ann Phys 339(3): 591-592

Filipe V et al (2010) Critical evaluation of nanoparticle tracking analysis (nta) by nanosight for the measurement of nanoparticles and protein aggregates. Pharm Res 27(5):796-810

Geers C et al (2016) A new angle on dynamic depolarized light scattering: number-averaged size distribution of nanoparticles in focus. Nano 8(34):15813-15821

Giesche H (1994) Synthesis of monodispersed silica powders in particle properties and reaction kinetics. J Eur Ceram Soc 14: 189-204

Giner-Casares JJ et al (2016) Inorganic nanoparticles for biomedicine: where materials scientists meet medical research. Mater Today 19(1):19-28 
Goodall DM (2010) Separation, size and charge determination of small molecules using ce in combination with uv area imaging. Chromatography Today December 31-34. http://www. chromatographytoday.com/article_read/830/

Hawe A et al (2011) Taylor dispersion analysis compared to dynamic light scattering for the size analysis of therapeutic peptides and proteins and their aggregates. Pharm Res 28(9): 2302-2310

Hulse W, Forbes R (2011) A taylor dispersion analysis method for the sizing of therapeutic proteins and their aggregates using nanolitre sample quantities. Int J Pharm 416(1):394-397

Laborda F et al (2016) Detection, characterization and quantification of inorganic engineered nanomaterials: a review of techniques and methodological approaches for the analysis of complex samples. Anal Chim Acta 904:10-32

Lattuada M, Hatton TA (2007) Functionalization of monodisperse magnetic nanoparticles. Langmuir 23(4):2158-2168

Merkus HG (2009) Particle size measurement: fundamentals, practice, quality. Springer, Dordrecht

Michen B et al (2015) Avoiding drying-artifacts in transmission electron microscopy: characterizing the size and colloidal state of nanoparticles. Sci Rep 5:9793

Moore TL et al (2015) Nanoparticle colloidal stability in cell culture media and impact on cellular interactions. Chem Soc Rev 44(17):6287-6305

Oukacine F et al (2015) Size-based characterization of nanoparticle mixtures by the inline coupling of capillary electrophoresis to taylor dispersion analysis. J Chromatogr A 1426:220-225
Park J et al (2004) Ultra-large-scale syntheses of monodisperse nanocrystals. Nat Mater 3(12):891-895

Pyell U et al (2015) Characterization of hydrophilic coated gold nanoparticles via capillary electrophoresis and taylor dispersion analysis. Part ii: Determination of the hydrodynamic radius distribution - comparison with asymmetric flow field-flow fractionation. J Colloid Interface Sci 457:131-140

Quinten, M. (2011) Optical properties of nanoparticle systems: Mie and beyond, Wiley-VCH Verlag $\mathrm{GmbH}$, Weinheim

Saux TL, Cottet H (2008) Size-based characterization by the coupling of capillary electrophoresis to taylor dispersion analysis. Anal Chem 80(5):1829-1832

Taylor G (1953) Dispersion of soluble matter in solvent flowing slowly through a tube. Proc R Soc Lond A Math Phys Sci 219(1137):186-203

Taylor G (1954) Conditions under which dispersion of a solute in a stream of solvent can be used to measure molecular diffusion. Proc R Soc Lond A Math Phys Sci 225(1163):473-477

Turkevich J et al (1951) A study of the nucleation and growth processes in the synthesis of colloidal gold. Discuss Faraday Soc 11(11):55-5\&

Wuelfing WP et al (1999) Taylor dispersion measurements of monolayer protected clusters: a physicochemical determination of nanoparticle size. Anal Chem 71(18):4069-4074 